\title{
A method for detecting undervalued resources with application to breeding birds
}

\author{
Daniel P. Shustack ${ }^{1}$ and Amanda D. Rodewald \\ School of Environment and Natural Resources, Ohio State University, \\ 2021 Coffey Road, 210 Kottman Hall, Columbus, Ohio 43210 USA
}

\begin{abstract}
Anthropogenic changes to ecosystems can decouple habitat selection and quality, a phenomenon well illustrated by ecological traps in which individuals mistakenly prefer low-quality habitats. Less recognized is the possibility that individuals might fail to select high-quality habitat because of the absence of some appropriate cue. This incorrect assessment of resource quality can lead to relatively high-quality resources being undervalued, whereby they support fewer individuals than optimal. We developed a habitat selection model to predict the expected patterns in patch-level density, fitness, and individual quality derived from either accurate assessment of habitat quality or from undervaluing of habitat patches (i.e., quality is not correctly assessed). Unlike previous habitat selection models, we explicitly and simultaneously incorporated variation in both individual and habitat quality into our estimates of realized fitness. Although multiple mechanisms can reduce patch-average density, fitness, and individual quality in less preferred patches, only undervaluation results in the occupation of higher-quality territories by similar-quality individuals in less preferred vs. preferred patches. We then looked for evidence of undervaluation in our seven-year data set of Acadian Flycatchers (Empidonax virescens) occupying forests in urbanizing landscapes in Ohio, USA. We suspected that forests within more urban landscapes may be undervalued in our study system because (1) urban forests typically support lower densities of Neotropical migratory birds than rural forests and (2) anthropogenic disturbance and habitat alterations are likely to result in mismatches between cues typically used in habitat selection and actual habitat quality. In contrast to our predictions, field data suggest that urban forests are not undervalued. Our work not only expands upon previous habitat selection models by considering undervaluation, but also demonstrates how predictions derived from our model can be tested using a long-term empirical data set.
\end{abstract}

Key words: Acadian Flycatcher; ecological trap; Empidonax virescens; fitness; habitat quality assessment; habitat selection; ideal free distribution; ideal preemptive distribution; individual quality; Neotropical migratory bird; undervalued resource; urban ecology.

\section{INTRODUCTION}

Understanding the mechanisms that generate patterns in the distribution of organisms is one of the central goals of ecology. As such, a variety of habitat selection models have been developed, tested, and refined with the ultimate aim of elucidating these mechanisms. The most commonly invoked models of habitat selection assume that individuals can accurately perceive, assess, and respond to habitat quality. One such model is the ideal free habitat selection model (Fretwell and Lucas 1970), which predicts that low-quality patches have lower densities than higher-quality patches. However, due to density-dependent mechanisms (e.g., crowding effect), individuals are expected to achieve equivalent levels of

Manuscript received 16 July 2009; revised 4 December 2009; accepted 24 December 2009. Corresponding Editor: J. M. Marzluff.

${ }^{1}$ Present address: Massachusetts College of Liberal Arts, Biology Department, 375 Church Street, North Adams, Massachusetts 01247 USA.

E-mail: Daniel.Shustack@mcla.edu fitness, irrespective of patch quality. Scaling this to the patch level, the average fitness of individuals in each patch (hereafter termed patch-average fitness) is predicted to be equal across patches of differing quality. Under ideal despotic habitat selection, lower habitat quality also leads to lower densities in those habitats but with one important qualification: because organisms defend territories for exclusive use, density-dependent feedbacks are dampened. Thus, the ideal despotic model predicts reduced patch-average fitness in patches of lower quality.

Accurate assessment of habitat quality is a key assumption of many habitat selection models. Yet, much empirical research suggests that accurate assessment of habitat quality is not ubiquitous, particularly amid anthropogenic disturbance and habitat modification (Schlaepfer et al. 2002). For instance, ecological traps arise where cues for habitat selection are disassociated from the underlying habitat quality (Ward and Schlossberg 2004, Ahlering and Faaborg 2006, Campomizzi et al. 2008). In ecological traps, individuals 
are drawn to habitats where they will ultimately experience low fitness. Conversely, high-quality habitat might be avoided due to a selection bias. For example, the removal or manipulation of a cue for habitat quality could prompt individuals to avoid high-quality habitats and, consequently, could result in lower densities in the less-preferred but high-quality habitat. Failure to select high-quality habitat would thereby lead to missed opportunities for fitness gains, lower population levels, and undervaluation of the avoided high-quality habitat (Gilroy and Sutherland 2007).

Although habitat selection affects an organism's fitness prospects, fitness is not entirely determined by the choice of habitat. Fitness (as measured by various aspects of reproductive success and survival) also can be a function of individual quality. Individual quality, in turn, is influenced by a variety of behavioral, physiological, and morphological attributes (Blackmer et al. 2005), is difficult to define (Moyes et al. 2009), and may vary with context (McNamara and Houston 1996).

If we consider that organisms do not always choose the highest-quality habitat and, further, that fitness is a function of both habitat and individual quality, then a direct comparison of patch density and patch-average fitness may reveal neither patch quality nor the mechanism of habitat selection. Although ecologists have long recognized that density may not accurately reflect habitat quality (Van Horne 1983), most generally assume that lower patch-average fitness reflects lower habitat quality (Johnson 2007). We suggest that an interaction between undervaluation of resources and variation in individual quality is another possible mechanism that should be considered.

Our curiosity was piqued by our long-term study of the forest-breeding Acadian Flycatcher (Empidonax virescens) across an urban to rural landscape gradient. Apparent in this population are two patterns suggestive of lower-quality habitat in urban compared to rural forests. Specifically, our previous research shows that (1) densities of Acadian Flycatchers are negatively related to the degree of urbanization in the landscape surrounding the forest patches (Rodewald and Bakermans 2006) and (2) many (but not all) fitness components are negatively related to the degree of urbanization (Rodewald and Shustack 2008, Shustack 2008). These and similar results (Bakermans and Rodewald 2006, Burhans and Thompson 2006, Grandmaison and Niemi 2007, Chamberlain et al. 2009) are typically thought to stem from the fact that urban habitats are lower in quality than more rural habitats. However, these interpretations fail to consider either the influence of individual quality on patch-average fitness or the possibility that resources are being undervalued.

We considered two hypotheses to explain the patterns we observed (i.e., lower density and lower patch-average fitness in one patch or habitat type vs. another). First, the "low-quality habitat" hypothesis posits that real differences in habitat quality exist among patches and individuals correctly select territories based on accurate information about the habitat quality. Consequently, we find lower densities in low-quality patches due to settlement choices, and we find lower patch-average fitness because of the comparably poorer resources. In contrast, the "undervalued resource" hypothesis supposes that despite similar quality of habitat, one patch (or habitat type) is selected in preference to another patch. In this case, behavior alone drives lower densities in certain patches. Moreover, because the highest-quality individuals will secure their preferred habitats, then a disproportionate number of lower-quality individuals should settle in less dense patches. If we assume that fitness is partly a function of individual quality, patches with low densities could have lower patch average-fitness compared to more densely occupied patches.

Our goal was to develop a habitat selection model that incorporated variation in individual quality and perception of the habitat or resource quality. To do this, we modified the Pulliam and Danielson (1991) ideal preemptive model of habitat selection to include variation of individual quality and errors in perception of habitat quality. We then parameterized our model in a simulation in order to identify a critical prediction that could be used to distinguish between the two hypothesized habitat selection mechanisms. Finally, we tested this critical prediction using our seven-year study of Acadian Flycatchers in central Ohio, USA.

\section{Methods}

\section{Model development}

We began with the ideal preemptive model of habitat selection proposed by Pulliam and Danielson (1991), developing our model in an aspatial two-patch system. Although this was an oversimplification of reality, it provided the necessary complexity to investigate the mechanisms we were exploring. This model is similar to the ideal free model proposed by Fretwell and Lucas (1970), in that individuals are ideal and have complete knowledge of the quality of the habitats. In both models, individuals are free of any costs associated with selecting habitats. However, the ideal preemptive model differs from the ideal free model in one key respect: it incorporates within-patch habitat heterogeneity so that each successively colonizing individual occupies a successively lower-quality territory without reducing the fitness prospects of the previous colonizers. There is no densitydependent reduction in habitat quality (i.e., through crowding), but rather each successive individual acquires a lower-quality territory because the highest-quality territories are settled first (i.e., territory or site dependence, as described by Rodenhouse et al. 1997). Thus, an individual can only choose a territory from among unoccupied territories because occupied territories have been "preempted." A key prediction of the ideal preemptive model is that individuals in the lowest-quality occupied territory in each patch will have equal (or nearly equal) fitness. This pattern occurs because colonizers will 
always select the best of the vacant territories from among all patches. A second prediction of the ideal preemptive model is that the patch-average fitness will be lower in the lower-quality patch. These predictions contrast with the ideal free distribution, in which the patch-average fitness is expected to be equal across patches. Further, the ideal preemptive model does not assume any resistance to subsequent settlers, as does the ideal despotic (dominance) model (Fretwell and Lucas 1970).

We defined $\beta_{\text {in }}\left(n_{i}\right)$ as the territory quality experienced by the individual that utilizes the $n$th best territory in habitat $i$ when there are $n_{i}$ individuals already breeding in habitat $i$ (following Pulliam and Danielson 1991). In contrast to the ideal preemptive model, individuals do not make their habitat selection decision based on $\beta_{\text {in }}\left(n_{i}\right)$. In order to incorporate the possibility of errors in the perception of habitat quality, as suggested by Gilroy and Sutherland (2007) where high-quality habitat is undervalued, we incorporated an additional term $\operatorname{Per}_{i}$ reflecting a modification to the perceived quality of habitat $i$. Thus, in our model, birds have information about the perceived quality of each territory, $\operatorname{Per}_{i} \beta_{\text {in }}\left(n_{i}\right)$, and choose an available territory that has the highest perceived quality. Although various relationships between perceived and actual habitat quality are possible (e.g., Kristan 2003), we used this simple multiplicative relationship to reflect the idea that all territories in a patch are not perceived at their actual quality because of a lack of some cue. Yet, the fitness of the individuals is related to the actual territory quality, $\beta_{\text {in }}\left(n_{i}\right)$.

A key assumption of previous habitat selection models (Fretwell and Lucas 1970, Pulliam and Danielson 1991, Rodenhouse et al. 1997, Donovan and Thompson 2001, Kokko and Sutherland 2001, Kristan 2003) is that all individuals are equal. Our model incorporated variation in individual quality that affects the fitness outcome of that individual. We assumed that individuals of equal quality will have equal fitness if they occupy territories of equal quality. However, if individuals of differing quality occupy territories of the same quality, the lower-quality individual will have lower fitness because of its lower individual quality. We defined $Q_{i}$ as the individual quality of individual $i$.

Each individual in the population selects a vacant territory that has the maximum perceived quality, $\operatorname{Per}_{i} \beta_{\text {in }}\left(n_{i}\right)$. Individuals select territories in descending sequence of individual quality, $Q_{i}$. Thus the highestquality individual selects the territory with the highest perceived quality and so on until all individuals have selected the available territory with the highest perceived quality at the time of their selection. Each individual will then experience a fitness that is related to its territory quality (i.e., $\left.\beta_{\text {in }}\left(n_{i}\right)\right)$ and individual quality (i.e., $Q_{i}$ ). We did not know the empirical relationship between the contribution of habitat quality and individual quality to fitness of an individual. Therefore we assumed that the fitness realized by an individual is the sum of the individual quality $\left(Q_{i}\right)$ and the habitat quality $\left(\beta_{\text {in }}\left(n_{i}\right)\right)$, with an additional term $(q)$ to define the relative contribution of individual quality as compared to habitat quality. Therefore the fitness of individual $i$ in territory $n$ in patch $i$ is given as $F_{i}=q Q_{i}+\beta_{\text {in }}\left(n_{i}\right)$. Thus, an average individual (i.e., $Q_{i}=0$ ) would have a fitness $\left(F_{i}\right)$ based solely on the habitat quality and aboveaverage individuals (i.e., $Q_{i}>0$ ) do better than average individuals and so on. We note that other relationships are possible and further research is needed to specifically describe the relative contributions of habitat quality and individual quality to fitness. However, this model provides a starting point for conceptually incorporating individual and habitat quality into fitness.

\section{Simulation}

We used this model to simulate the two hypotheses that we suspected could produce lower densities and lower patch-average fitness in one patch vs. another. Although we used a simple two-patch case, we expected the results to apply in any system where habitats are heterogeneous in quality or perceived quality. Each patch (arbitrarily denoted with subscripts $U$ and $R$ reflecting our interests in urban and rural patches) had 100 territories that were assigned integer habitat quality values based on the exponential function $f(x)=\theta e^{-\theta x}$ (Pulliam and Danielson 1991, Kristan 2003), with $x=0$ to 10 by integers. This generated a habitat distribution in which low-quality territories were more abundant, higher-quality territories were fewer, and the relative number of each was determined by $\theta$.

We generated values of individual quality $\left(Q_{i}\right)$ for a population $(N)$ of 100 individuals from a normal distribution of mean $=0$ and $\mathrm{SD}=5$. We used a standard deviation of five so that approximately $95 \%$ of the individuals would have a $Q_{i}$ between -10 and +10 . We assumed that the individual quality contribution $(q)$ to fitness was less than (e.g., $q=0.5$ ) the contribution of the habitat quality, reflecting the greater importance of habitat quality over individual quality to fitness. Further, we bound the fitness $\left(F_{i}\right)$ of each individual between 0 and 10 in order to reflect maximum and minimum thresholds on fitness. For example, an individual with $Q_{i}=10$ will have $F_{i}=5$ when $\beta_{\text {in }}\left(n_{i}\right)=$ 0 , but will have $F_{i}=10$ when $\beta_{\text {in }}\left(n_{i}\right) \geq 5$. In order to garner qualitative predictions from these models, it was not necessary to explicitly define "fitness." For this specific parameterization, fitness could simply be thought of as the number of offspring produced by the individual in that territory. In other cases, survival, mass gain, or other reproductive metrics might be the relevant fitness components under investigation. Rather than generating specific quantitative outputs, the purpose of the simulation was to elucidate qualitative predictions that might separate our two hypotheses.

Each simulation consisted of placing individuals onto territories based on the decision rules of the model (i.e., selecting the perceived best available territory from among all patches) and subsequently calculating the 
fitness of each individual based on the territory quality and the individual quality. The output that we collected from each simulation consisted of (1) the number of individuals in each patch, (2) the patch-average quality of individuals and the patch-average fitness of each patch, and (3) the average quality and fitness of the average individuals within each patch. For this calculation, we defined individuals of "average" quality as individuals with $\left|Q_{i}\right|<0.25 \mathrm{SD}$. We performed each simulation 100 times. We report the mean values from the 100 runs and their standard deviations. We recorded this quantitative output to determine if our hypotheses could generate the observed patterns of density and patch-average quality and if we could derive qualitative predictions that might help to separate our two hypotheses.

We simulated the two hypotheses by altering parameters $\theta$ and Per. First we simulated the "low-quality habitat" hypothesis, which assumes that habitat quality is lower in one patch and that assessment of habitat quality is accurate (Fig. 1A). We made $\theta_{\mathrm{R}}=0.1, \theta_{\mathrm{U}}=$ $0.4, \operatorname{Per}_{R}=1$, and $\operatorname{Per}_{U}=1$. Next we simulated the "undervalued resource" hypothesis, which states that despite similar habitat quality in both patches, individuals perceive one habitat at a lower quality than it actually is (Fig. 1B). For this hypothesis we made $\theta_{\mathrm{R}}=$ $0.1, \theta_{\mathrm{U}}=0.1, \operatorname{Per}_{\mathrm{R}}=1$, and $\operatorname{Per}_{\mathrm{U}}=0.5$ (Table 1). Rather than generating quantitative predictions, which would necessitate sensitivity analysis, we aimed only to identify key qualitative predictions that could serve to distinguish between the two hypotheses.

\section{Field methods}

Our ongoing research with Acadian Flycatchers proved to be an excellent system in which to test our hypotheses. Our prior work showed that Acadian Flycatchers occupied patches at lower densities, exhibited higher turnover rates, produced fewer fledglings over a breeding season, and initiated clutches later in the spring in forested sites with greater amounts of urbanization in the surrounding landscape (Bakermans and Rodewald 2006, Rodewald and Bakermans 2006, Rodewald and Shustack 2008, Shustack and Rodewald 2010). Thus, this population of Acadian Flycatchers in an urban to rural landscape gradient displayed the patterns that could be produced by either of our hypotheses.

The methods we used for quantifying the landscape around each site and collecting field data are described in detail in a previous study (Rodewald and Shustack 2008). Briefly, within a circular $1 \mathrm{~km}$ radius area centered on each study site, we calculated proportions of different land cover types and the number of buildings by inspection of digital orthophotos (20022004). A principal components analysis performed on five variables describing development in the landscape produced a first principal component that explained $80 \%$ of the variation among sites (eigenvalue $=3.99$ ). We hereafter refer to this first component as the "urban index" (Rodewald and Shustack 2008), where higher values reflected sites in more urbanized landscapes. The urban index loaded positively for the number of buildings (0.92), percentage of cover by roads (0.94), pavement $(0.90)$, and lawn (0.88), but loaded negatively for percentage of cover by agriculture $(-0.83)$.

As possible, individual Acadian Flycatchers were measured, banded, and monitored through their breeding cycle in each year. We recorded tarsus length, unflattened wing chord, and mass for individual birds. Based on intensive field methods (see Shustack and Rodewald 2010), we located nests and determined when clutches were initiated. We standardized the clutch initiation dates across years to the median clutch initiation date in 2006 (e.g., in 2005 the median clutch initiation date was two days later than in 2006; therefore we subtracted two days from each clutch initiation date from 2005). We tracked the number of fledglings produced by each individual over the course of the breeding season and the number of nesting attempts by each individual. In each year we intensively searched the study sites to determine if previously banded individuals returned to the study site.

\section{Model application}

Based on the simulation results (see Results) we identified one critical prediction that differentiated between our two hypotheses. When comparing individuals of equal quality (i.e., $\left|Q_{i}\right|<0.25 \mathrm{SD}$ ) from each habitat, fitness was equal under the "low-quality habitat" hypothesis. However, under the "undervalued resource" hypothesis, the fitness of the individual in the less preferred patch was higher than that of an individual of similar quality in the preferred patch.

Based on this critical difference, we examined relationships among individual quality and fitness components in our population of Acadian Flycatchers. We considered our "undervalued resource" hypothesis to be supported, if when comparing individuals of similar quality, we were to find that urban birds had higher fitness than birds in rural forests. On the other hand, if fitness of individuals with equal quality were similar regardless of position along the urban index, the "low-quality habitat" hypothesis would be supported.

Although our conceptual model (i.e., Fig. 1) was nonspecific with regard to individual quality and fitness components, the testing of the critical prediction with field data required specific parameterization. We do not know which attributes contribute to or are correlated with individual quality in the Acadian Flycatcher, but morphological features, at least in part, are likely candidates for flycatchers (Murphy 2007). Further, based on our previous work (Shustack 2008, Shustack and Rodewald 2010), we have evidence that morphology in Acadian Flycatchers is related to at least some aspects of fitness. Despite the fact that our prior work limited the fitness components and morphological variables examined, we still found that smaller, lighter, and shorter-winged females tended to initiate egg-laying 

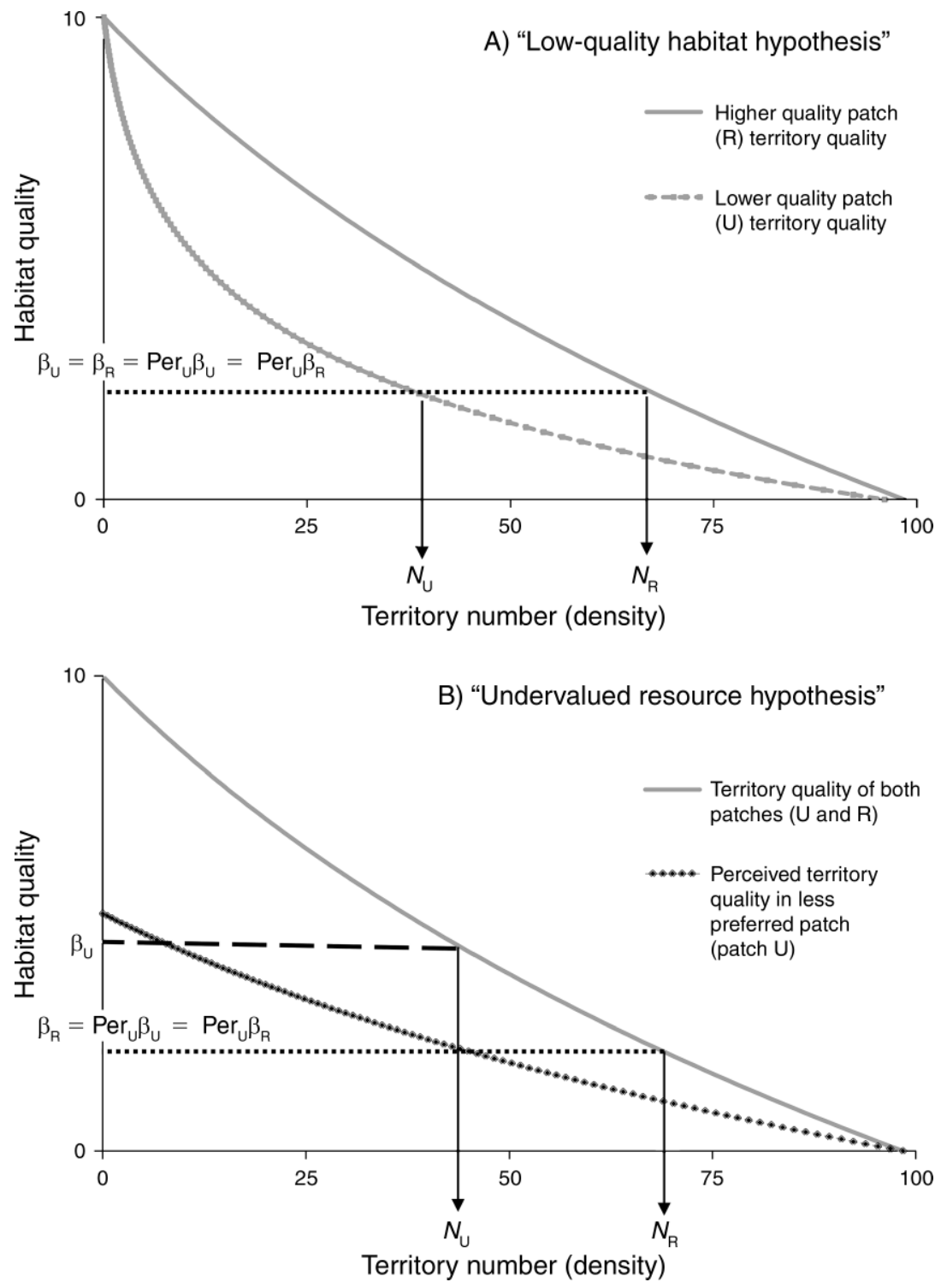

FIG. 1. Territories are ordered and numbered separately for each patch, here labeled urban (U) and rural (R) for illustration purposes, in order of descending territory quality. In both panels (A) and (B), individuals select the available territory with the best perceived quality. However, in panel (A), the "low-quality habitat" hypothesis, the perceived territory quality (Per $\beta$ ) is equal to the actual territory quality $(\beta)$, but territories in patch $\mathrm{U}$ are of lower quality ( $\beta$, dashed curved line) than in patch $\mathrm{R}$ (solid curved line). Thus, individuals sequentially select territories from the best available territories, resulting in higher densities in the patch $\mathrm{R}\left(N_{\mathrm{R}}\right)$ than patch $\mathrm{U}\left(N_{\mathrm{U}}\right)$. The horizontal dotted line shows the final densities (i.e., $N=100$ total individuals) and territory quality of the last individuals to select a territory from either patch U and R. In panel (B), the "undervalued resource" hypothesis, habitat quality is similar between patches $\mathrm{R}$ and $\mathrm{U}$ (solid upper line), but the perceived territory quality in patch $\mathrm{U}\left(\mathrm{Per}_{\mathrm{U}}\right)$ is lower $\left(\mathrm{e} . \mathrm{g}\right.$., $\mathrm{Per}_{\mathrm{U}}=0.5$ in this example; see Methods: Model development). Individuals sequentially select territories based on the perceived quality so that final distribution (the lower dotted line, when $N=100$ total individuals) contains more individuals in patch $\mathrm{R}\left(N_{\mathrm{R}}\right)$ than in patch $\mathrm{U}$ $\left(N_{\mathrm{U}}\right)$, but the last individuals to select each site experience similar perceived territory quality, yet the individual in patch $\mathrm{U}$ experiences actual territory quality equal to $\beta_{\mathrm{U}}$ (i.e., the upper long-dashed line).

later in the spring. We recognize that other traits, such as age (Nol and Smith 1987, Preault et al. 2005), personality, and behavior, also relate to individual quality (Biro and Stamps 2008) and that these correlates of fitness may vary temporally or spatially (Duckworth 2008). Unfortunately, we do not have data on these traits, and consider individual morphology a reasonable starting point for testing our hypotheses with our field data. Although morphological measurements are plausible metrics for individual quality in this case, in other study systems it may be more appropriate to use different metrics for individual quality.

To test our field data for the critical prediction separating our two hypotheses, for both males and 
TABLE 1. Parameter inputs and summary outputs for two simulated hypotheses to account for distributions of birds across urban and rural patches.

\begin{tabular}{|c|c|c|c|c|c|c|c|c|c|c|c|c|}
\hline \multirow[b]{3}{*}{ Parameter } & \multicolumn{6}{|c|}{ Low quality habitat } & \multicolumn{6}{|c|}{ Undervalued resources } \\
\hline & \multicolumn{2}{|c|}{ Rural } & \multicolumn{2}{|c|}{ Urban } & \multicolumn{2}{|c|}{$t$-test $P$ value } & \multicolumn{2}{|c|}{ Rural } & \multicolumn{2}{|c|}{ Urban } & \multicolumn{2}{|c|}{$t$-test $P$ value } \\
\hline & Mean & SD & Mean & SD & Mean & SD & Mean & SD & Mean & SD & Mean & SD \\
\hline$\theta$ & 0.100 & $\mathrm{n} / \mathrm{a}$ & 0.400 & $\mathrm{n} / \mathrm{a}$ & & & 0.100 & $\mathrm{n} / \mathrm{a}$ & 0.100 & $\mathrm{n} / \mathrm{a}$ & & \\
\hline Per & 1.000 & $\mathrm{n} / \mathrm{a}$ & 1.000 & $\mathrm{n} / \mathrm{a}$ & & & 1.000 & $\mathrm{n} / \mathrm{a}$ & 0.500 & $\mathrm{n} / \mathrm{a}$ & & \\
\hline$N$ & 65.430 & 1.305 & 34.570 & 1.305 & & & 61.00 & 0.000 & 39.00 & 0.000 & & \\
\hline$N_{\text {ave }}$ & 6.480 & 1.667 & 13.160 & 2.863 & & & 10.710 & 2.249 & 8.590 & 2.738 & & \\
\hline$F$ & 5.754 & 0.167 & 3.496 & 0.231 & 0.008 & 0.013 & 6.305 & 0.175 & 5.659 & 0.240 & 0.272 & 0.131 \\
\hline$F_{\text {ave }}$ & 4.783 & 0.321 & 4.684 & 0.401 & 0.580 & 0.265 & 4.417 & 0.428 & 8.856 & 0.293 & 0.005 & 0.008 \\
\hline$Q$ & 0.988 & 0.444 & -1.901 & 0.563 & 0.009 & 0.013 & 2.112 & 0.541 & -3.211 & 0.577 & 0.000 & 0.000 \\
\hline$Q_{\text {ave }}$ & 0.055 & 0.204 & -0.010 & 0.278 & 0.580 & 0.265 & -0.026 & 0.282 & 0.043 & 0.282 & 0.450 & 0.329 \\
\hline
\end{tabular}

Notes: For both low-quality habitat and undervalued resources hypotheses, overall $N=100$ and $q$, the relative contribution of individual quality vs. habitat quality, is 0.500 ; the $\mathrm{SD}$ of $q$ is 5.000 . The value $\theta$ defines the habitat quality function based on $f(x)=$ $\theta e^{-\theta x}$ and Per is a term defining perception of habitat quality; SD is not applicable (n/a). Using these inputs, the simulation was run 100 times. $N$ is the average number of individuals; $N_{\text {ave }}$ is the average number of individuals that had $\left|Q_{i}\right|<0.25 ; F$ is patch-average fitness; $Q$ is patch-average individual quality; $F_{\text {ave }}$ is the average fitness of individuals with $\left|Q_{i}\right|<0.25 \mathrm{SD}$; and $Q_{\text {ave }}$ is the average quality of individuals with $\left|Q_{i}\right|<0.25 \mathrm{SD}$. Following each simulation, the $P$ values from nonparametric $t$ tests between urban and rural means for $F, F_{\text {ave }}, Q$, and $Q$ ave were calculated; the average $P$ value of the $100 t$ tests and SD of the $100 P$ values are reported. For each simulation the slope estimate for a patch effect on the relationship between fitness and bird quality was determined: for the low-quality habitat hypothesis, the average slope estimate is $-0.110(P=0.480)$; for the undervalued resources hypothesis, the slope estimate is $3.724(P<0.0001)$.

females separately, we looked for relationships between individual morphology (wing length, tarsus length, body size, body mass, and condition) and fitness components (clutch initiation date, nest survival, number of fledglings, probability of renesting, and probability of being resighted in a subsequent year) after accounting for habitat quality (i.e., urbanization). Specifically, we used regression models with individual morphology and the urban index as explanatory variables to predict each fitness variable. We reasoned that if the "low-quality habitat" hypothesis were the mechanism generating the distribution of Acadian Flycatchers in our study system, then there should be a nonsignificant parameter estimate for the urban index (representing habitat type in our model) equal to zero. Parameter estimates for the urban index that are statistically significant and in the direction suggestive of lower-quality individuals having higher fitness component values would support the "undervalued resource" hypothesis.

Because we had data on only some components of Acadian Flycatcher fitness and had no a priori reason to favor certain components over others, we decided to consider all fitness components. For some individuals, we had more than one year of fitness-related data (i.e., they were confirmed breeders in more than one year), but typically individuals were only captured once. Consequently, we used morphological data from initial captures and fitness data from all years. Fitness information from each female or male from each year was treated as an independent observation in the analyses.

In our previous research we reported relationships between some individual morphology variables and the urban index (Rodewald and Shustack 2008). High levels of multicollinearity could obscure the effects of the urban index and the individual morphological metrics (Gotelli and Ellison 2004). We assessed possible collinearity between the urban index and all individual morphological measurements (each sex separately) by using Spearman's correlations. All correlations were low $(r<0.25)$; only the correlations between the urban index and male wing chord and male body mass had all $P<0.05$. We therefore did not consider multicollinearity to be a concern in our analyses.

We used different model structures relating fitness and morphometrics as appropriate. Because Poisson regressions did not converge properly for the number of fledglings, we used negative binomial regression (PROC GENMOD, SAS Institute 2002) to examine the relationship between the number of fledglings and individual morphological variables and the urban index (Allison 1999). For both Poisson and negative binomial regressions, we report $\hat{c}$, a measure of overdispersion. For the negative binomial regressions, we also report the dispersion parameter. We applied least squares regression to examine relationships between the natural logarithm of the clutch initiation date and individual morphological variables and the urban index. We used the natural $\log$ of the clutch initiation date to improve the normality of residuals. Although residuals from one regression were still non-normal, for simplicity we used this transformation for all of these regressions. (Non-normality should not affect the direction of the slope parameter, the object of our main interest.) We report the $P$ value for ShapiroWilks tests for normality for these regressions (PROC UNIVARIATE). We used logistic exposure models (PROC GENMOD; Shaffer 2004) to examine the relationship between nest survival and individual morphological variables and the urban index. In the nest survival regressions, we included the day of the year as a variable because our previous results have indicated an important seasonal effect to nest survival (D. Shustack, unpublished data). We used logistic regression (PROC LOGISTIC) to assess the probability of renesting and 
resighting an individual in a future year based on individual morphology and the urban index. We assessed the goodness of fit of the logistic regression and logistic exposure models using Hosmer-Lemeshow tests (Hosmer and Lemeshow 1989). We used Poisson regressions (PROC GENMOD) to relate the number of nesting attempts to individual morphological variables and the urban index. Because the Poisson regressions were underdispersed $(\hat{c} \approx 0.3)$, we scaled the standard error and significance estimates by the square root of $\hat{c}$ (Allison 1999; PSCALE option in SAS). Although we recognize that numerous significance tests of the slopes of parameters in our models were performed, we chose this approach in order to preserve statistical power (Nakagawa 2004). We report nominal $P$ values throughout.

\section{Results}

\section{Model results}

Our simulations confirm our qualitative predictions that both hypothesized mechanisms are capable of generating the common patterns reported from urbanizing landscapes. Both mechanisms produced lower densities, lower patch average fitness, and lower patch average individual quality in the lower-quality patch compared to the higher-quality patch. However, comparing the average individuals (i.e., $\left|Q_{i}\right|<0.25 \mathrm{SD}$ ) across sites revealed a critical difference. Average individuals under the "low-quality habitat" hypothesis had similar fitness regardless of which patch they occupied. In contrast, with the "undervalued resource" hypothesis, average individuals in the less preferred patch had higher fitness than the average individual in the preferred patch. Thus, for a given level of individual quality, individuals in the less preferred patch had higher fitness than individuals in the preferred patch (despite equality in patch quality; Table 1, Fig. 2). Considering two individuals (e.g., A and B) of similar quality (e.g., $Q_{\mathrm{A}} \approx Q_{\mathrm{B}}$ ) reveals why this pattern emerged. Because individuals $\mathrm{A}$ and $\mathrm{B}$ are of similar quality, the term $q Q_{i}$ contributes equally to $F_{i}$ for both individuals. Therefore only habitat quality, $\beta_{\mathrm{A}}$ and $\beta_{\mathrm{B}}$, leads to variation in their fitness. Under either hypothesis, if both individuals went to the same patch, they would occupy territories of approximately equal quality and would attain similar fitness levels. If individual A and B settled in different patches, both would select territories with similar perceived quality. Under the "low-quality habitat" hypothesis, the perceived habitat quality is synonymous with the actual habitat quality $\left(\operatorname{Per}_{R}=\operatorname{Per}_{U}\right)$. Therefore individuals $\mathrm{A}$ and $\mathrm{B}$ would occupy territories so that $\beta_{\mathrm{A}}$ $\approx \beta_{B}$; thus individuals $A$ and $B$ would have similar fitness (horizontal dotted line in Fig. 1A). Under the "undervalued resource" hypothesis, the individual that selected the less preferred patch actually selected a territory where $\operatorname{Per}_{U} \beta_{U} \approx \beta_{R}$. In other words, the individual in the less preferred patch actually selected a territory (i.e., horizontal dashed line in Fig. 1B) that is better than the territory available to the individual

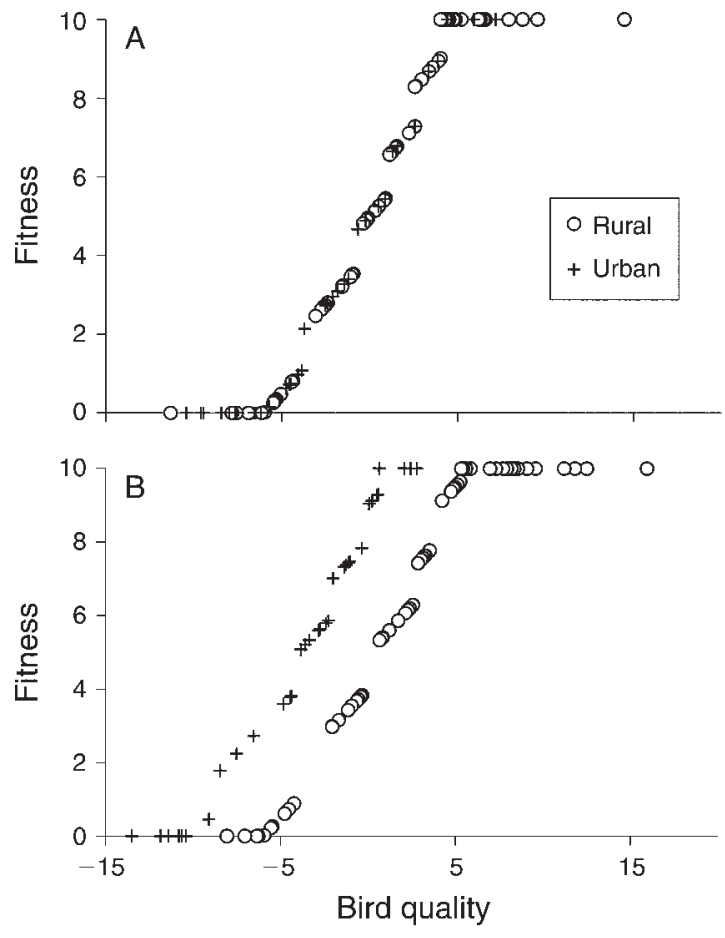

FIG. 2. Example output from one run of each simulation reflecting two hypotheses generating bird distributions. Notice that in panel (A), the "low-quality habitat" hypothesis, individuals of equal quality experience similar fitness values because they appropriately evaluate habitat quality and thus select available territory with the highest habitat quality, even though one patch (e.g., the urban patch) has more territories of lower quality. In panel (B), the "undervalued resource" hypothesis, individuals select territories based on perceived habitat quality, which is lower for the less preferred patch (e.g., the urban forest) even though both patches are of equal quality. Thus, individuals that select the less preferred patch (e.g., the urban patch) experience a territory quality higher than the perceived value, resulting in higher fitness for those individuals.

settling in the preferred patch, where the best territories were already occupied. In the case of our simulations where $\operatorname{Per}_{U}=0.5$, the individual selecting the territory from the preferred patch selected a territory that was equal to $\beta_{\mathrm{R}} / 0.5$, or two times better than the next available territory in the preferred patch.

\section{Model application}

When we accounted for individual quality, none of the negative binomial regressions relating the number of fledglings produced over the course of the breeding season had regression coefficients for the urban index that were significant at $P<0.05$. The three models (male tarsus, male condition, and male body mass) with all $P<0.1$ for the urban index all had slopes in the direction opposite that predicted by the "undervalued resource" hypothesis (Table 2; Appendix A). Three simple linear regressions (male condition, male wing. and male body mass) for the date of clutch initiation had significant $(P<0.05)$ slope estimates for the urban index, but here too, the direction of the slopes 
TABLE 2. Regression coefficients (with SE in parentheses) for the effect of urbanization (urban index) in models containing each morphology variable to predict the fitness components for an Acadian Flycatcher (Empidonax virescens) population nesting in central Ohio, USA, 2001-2007.

\begin{tabular}{|c|c|c|c|c|c|c|}
\hline $\begin{array}{l}\text { Morphology } \\
\text { variable }\end{array}$ & $\begin{array}{l}\text { No. seasonal } \\
\text { fledglings }\end{array}$ & $\begin{array}{l}\text { Clutch } \\
\text { initiation }\end{array}$ & Nest survival & $\begin{array}{c}\text { Probability of } \\
\text { renesting }\end{array}$ & $\begin{array}{c}\text { Probability of } \\
\text { resight }\end{array}$ & $\begin{array}{l}\text { No. nesting } \\
\text { attempts }\end{array}$ \\
\hline \multicolumn{7}{|l|}{ Female } \\
\hline $\begin{array}{l}\text { Tarsus } \\
\text { Body size } \\
\text { Condition } \\
\text { Wing } \\
\text { Mass }\end{array}$ & $\begin{array}{r}-0.087(0.22) \\
-0.109(0.23) \\
0.021(0.21) \\
-0.063(0.22) \\
0.028(0.21)\end{array}$ & $\begin{array}{r}0.006(0.01) \\
0.001(0.01) \\
0.012(0.01) \\
-0.004(0.01) \\
0.012(0.01)\end{array}$ & $\begin{array}{l}0.131(0.23) \\
0.091(0.23) \\
0.227(0.23) \\
0.09(0.22) \\
0.217(0.23)\end{array}$ & $\begin{array}{l}0.038(0.31) \\
0.012(0.32) \\
0.089(0.32) \\
0.021(0.32) \\
0.093(0.31)\end{array}$ & $\begin{array}{r}0.032(0.53) \\
-0.126(0.54) \\
-0.148(0.52) \\
-0.236(0.53) \\
-0.145(0.52)\end{array}$ & $\begin{array}{l}-0.028(0.09) \\
-0.011(0.09) \\
-0.056(0.09) \\
-0.013(0.09) \\
-0.058(0.09)\end{array}$ \\
\hline \multicolumn{7}{|l|}{ Male } \\
\hline $\begin{array}{l}\text { Tarsus } \\
\text { Body size } \\
\text { Condition } \\
\text { Wing } \\
\text { Mass }\end{array}$ & $\begin{array}{l}-0.198(0.11) \dagger \\
-0.178(0.11) \\
-0.206(0.11) \dagger \\
-0.165(0.1) \\
-0.193(0.11) \dagger\end{array}$ & $\begin{array}{l}0.012(0.01) \dagger \\
0.01(0.01) \dagger \\
0.013(0.01)^{*} \\
0.016(0.01)^{*} \\
0.015(0.01)^{*}\end{array}$ & $\begin{array}{l}0.058(0.12) \\
0.063(0.12) \\
0.057(0.12) \\
0.028(0.11) \\
0.037(0.11)\end{array}$ & $\begin{array}{l}0.156(0.17) \\
0.123(0.18) \\
0.215(0.18) \\
0.195(0.17) \\
0.254(0.17)\end{array}$ & $\begin{array}{r}-0.063(0.23) \\
-0.048(0.24) \\
0.042(0.24) \\
0.007(0.21) \\
0.004(0.21)\end{array}$ & $\begin{array}{l}-0.078(0.05) \dagger \\
-0.065(0.05) \\
-0.096(0.05)^{*} \\
-0.094(0.04)^{*} \\
-0.113(0.04)^{*}\end{array}$ \\
\hline
\end{tabular}

Notes: The significance level of the urbanization effect is indicated. Values in italics are regressions that align with the undervalued resources hypothesis; other values are regressions that align with the low-quality habitat hypothesis. Appendices A, B, and $\mathrm{C}$ contain complete regression results, including parameter estimates for the morphological variables and model fit information.

$* P<0.05 ; \dagger P<0.1$

was opposite that predicted by the "undervalued resource" hypothesis. Two other regressions (male tarsus and male body size) had all $P<0.1$ for the urban index, but these slopes were also in the direction opposite the "undervalued resource" hypothesis (Table 2; Appendix A). The urban index was not a significant regression coefficient for any of the regressions for nest survival (logistic exposure regression; Appendix B), probability of renesting (logistic regression; Appendix B), or probability of resighting individuals in subsequent years (logistic regression; Appendix C; all $P>0.10$ ). Three Poisson regressions (male condition, male wing, and male body mass) for the number of nesting attempts had parameter estimates for the urban index with all $P<0.05$, but again, the direction of the slopes was opposite that predicted by the "undervalued resource" hypothesis. The slope of the urban index had $P<$ 0.1 in one other model (male tarsus), but the slope was also opposite that predicted by the "undervalued resource" hypothesis (Table 2; Appendix C).

\section{DisCUSSION \\ Modeling}

We developed a habitat selection model that explicitly incorporated variation in individual quality and errors in assessment of habitat quality. With this model we demonstrated that multiple mechanisms can generate patterns commonly observed in nature. Specifically, our model shows that lower density and lower patch-level fitness can result from multiple processes and do not necessarily indicate that one patch is of lower quality than another, contrary to the typical interpretation of these patterns. Although low-quality habitat can generate these patterns (i.e., the "low habitat quality" hypothesis), so too can a situation in which one habitat is undervalued (e.g., Gilroy and Sutherland 2007). The disconnection between habitat quality and fitness and density measures can arise when individuals fail to recognize and select high-quality habitats, combined with variation in individual quality that contributes to fitness (e.g., the "undervalued resource" hypothesis). These results suggest that density and reproductive output alone are not sufficient to distinguish among mechanisms underlying the patterns; additional criteria are needed.

Output from our simulation models revealed the key piece of information needed to distinguish between the "low-quality habitat" and "undervalued resource" hypotheses: namely, individual quality. If individuals of similar quality occupying different habitats experience similar levels of fitness, the low-quality habitat hypothesis is supported. Alternatively, if the individual in the less preferred, lower-density habitat experiences higher fitness than a similar-quality individual in the preferred, higherdensity habitat, then the "undervalued resource" hypothesis triumphs. In this way, our models establish a clear qualitative and conceptually simple prediction to distinguish these two mechanisms. We recognize that this simplicity stops short of the field, as the application of our models to empirical data is limited to studies that have metrics of both fitness and individual quality. The approach also requires that fitness and individual quality are linked within the focal organism. Nevertheless, the application of our model may prove increasingly useful as researchers continue to examine relationships between individual quality and fitness.

\section{Model application}

For illustrative purposes, we applied our model to seven years of field data on the Neotropical migratory Acadian Flycatcher, a species for which density and reproductive output are lower in urban than rural forests. Finding no apparent effect of habitat quality (i.e., urbanization) on the fitness of individuals of similar quality, we have little evidence to suggest that urban forests are being under- 
valued by this species. If anything, the significant parameter estimates in the opposite from predicted direction suggest that Acadian Flycatchers might be slightly overvaluing urban forests, a situation reminiscent of ecological traps (Gates and Gysel 1978). As a whole, our data support the notion that forests in more urban areas are indeed low-quality habitat for Acadian Flycatchers and that the flycatchers appropriately avoid these habitats, thus leading to lower densities reported in these areas (Rodewald and Bakermans 2006). These results support our previous suggestion (Rodewald and Shustack 2008, Shustack and Rodewald 2010) that lowerquality individuals may be relegated to these urban habitats, either through competitive exclusion in a despotic manner from higher-quality rural forests or because they naively select these low-quality forests or are unable to select better forests.

One of the key assumptions underlying our specific application of this model is that fitness can be predicted from morphological traits. Despite our evidence of relationships between morphological and fitness attributes used in our analysis, other measures may be equivalent or better indicators of quality for Acadian Flycatchers. In general, little is known about which factors contribute to variation in individual quality in birds and even less for Acadian Flycatchers. Further complicating the issue is that the morphological features contributing to fitness may vary among years (Grant 2003) and any given trait could potentially have opposing effects on different fitness components, as seen in our Acadian Flycatchers (Table 2; Appendices A, B, and C). The lack of information regarding which attributes constitute individual quality may be one reason why individuals are often assumed to be equal in modeling studies (Fretwell and Lucas 1970, Pulliam and Danielson 1991, Rodenhouse et al. 1997, Donovan and Thompson 2001, Kokko and Sutherland 2001, Kristan 2003). Nevertheless, in wild populations, some aspects of fitness are often related to morphology: bill size (Gosler 1987 [foraging efficiency], Grant 2003 [survival]); bird size (Schluter and Smith 1986 [overwinter survival], Neto and Gosler 2005 [egg volume], Murphy 2007 [lifetime reproductive success and clutch size]); genetic composition (Ortego et al. 2007, Tomiuk et al. 2007 [clutch size and laying date]); foraging ability and nest attentiveness (Lewis et al. 2006); personality (Biro and Stamps 2008); and aggression (Duckworth 2008). For example, personality traits such as boldness and aggression may improve nest survival by discouraging nest predation (Olendorf and Robinson 2000, Jonart et al. 2007).

Our model held that territories were occupied by successively lower-quality individuals in decreasing order of (perceived) quality of territories. This relationship might arise in at least two ways. Individuals of higher quality may arrive in advance of lower-quality individuals, and thus select higher-quality territories (Smith and Moore 2005). Earlier arrival by higherquality individuals might be achieved due to those individuals overwintering on better habitats or making faster northward migration (Marra et al. 1998). Another mechanism whereby successively lower-quality individuals occupy lower-quality territories is by direct competition or fighting (Jonart et al. 2007) for territories, irrespective of arrival time, whereby outcomes of competition sort territory occupants by individual quality. However, there may be instances in which individuals do not sort positively based on habitat quality. For instance, in ecological traps, the highestquality individuals may be selecting the lowest-quality territories. Indeed, once we consider that fitness is a result of both individual quality and habitat quality, it raises the possibility that ecological trap effects may be masked or dampened when high-quality individuals select low-quality territories. Clearly, this raises an important issue for future investigation.

We recognize that our approach may have limited applicability to systems with little or no variation in either individual quality or territory quality. In these cases, all variation in fitness metrics will be attributed to the parameter with variance. Moreover, low variance in either individual quality or habitat quality reduces the likelihood of detecting undervaluation due to statistical issues associated with small variances. If there is little variance in individual quality, then our model simplifies to the ideal preemptive model (Pulliam and Danielson 1991). If there is little variance in territory quality, then undervaluation of the resource (or the converse, ecological traps) cannot occur, by definition. Thus, one prerequisite for application of our model is variance in both territory and individual quality.

The application of our model also requires that specific components of fitness be selected amid the many possible variables or aspects of fitness, such as egg mass, clutch size, fledglings mass or size, fledgling survival, timing of fledging, recruitment, or foraging efficiency. We selected fitness components that are among the most commonly collected in demographic studies of birds, but there remains the possibility that other fitness metrics might have produced contrary results. Trade-offs in fitness components (Chalfoun and Martin 2007) and scale dependency (Pidgeon et al. 2006) add to the difficulty in testing the "undervalued resource" hypothesis.

As a whole, our results suggest that Acadian Flycatchers are fairly effective at resource-matching. Because the ability to perceive and select appropriate habitat has direct implications for survival and reproduction, there should be strong selective pressure for good decision-making. Acadian Flycatchers should have access to several indirect and direct indicators of habitat quality. First, because Acadian Flycatchers are highly mobile, they can visit multiple sites to assess key attributes, such as habitat structure, food availability, and risk of predation, as indirect cues of fitness prospects for that habitat. Second, Acadian Flycatchers adjust future decisions based upon their own 
performance in a current patch. However, we have previously shown that such passive demographic responses in a highly mobile songbird are unlikely to generate the observed pattern of lower densities of Acadian Flycatchers in urban areas (Shustack and Rodewald 2008). Third, flycatchers could assess the relative success of neighbors or other individuals and subsequently choose to settle in areas where others have demonstrated success (Doligez et al. 2002, Betts et al. 2008). The presence and reproductive success of other individuals (conspecific and heterospecific) should serve as a reliable cue for habitat quality. In fact, the density of other Neotropical migratory birds is the best predictor for Acadian Flycatcher density in our study system (Rodewald, in press).

Our study expands our understanding of the ecology of habitat selection in two important ways. First, our model demonstrates that perceptual errors leading to undervaluation of a habitat can, in fact, generate patterns of lower density and lower fitness, even in the absence of differences in habitat quality among sites. In this way, not only does our model affect the way in which we conceptualize landscapes, but it also provides a useful tool for evaluating undervaluation of habitats by considering the fitness of birds of similar quality across habitats. Second, we advance our understanding of urban ecology by providing strong empirical evidence that urban habitats in Midwestern landscapes are not undervalued and may indeed represent lower-quality habitats for Neotropical migrants. In light of this, we urge conservation biologists to continue research that seeks to identify and restore those habitat features that most strongly constitute habitat quality for sensitive and declining species in urbanizing landscapes.

\section{ACKNOWLEDGMENTS}

Funding for this research was provided by the National Science Foundation (DEB-0340879 and DEB-0639429 ADR), the Ohio Division of Wildlife, the Ohio Agricultural Research and Development Center, and The Ohio State University Swank Program in Rural-Urban Policy. We are most grateful to M. H. Bakermans, K. L. Borgmann, L. Kearns, F. V. L. Leston, J. R. Smith-Castro, and N. Sundell-Turner for their dedicated efforts in the field and laboratory. We thank the following technicians for their help in the field: T. Brown, M. Carll, J. Fullerton, A. Gilmore, B. Graves, J. Gray, S. Hazzard, A. Highland, L. Hitchcock, M. Howie, E. Interis, T. Jones, M. Kanode, J. Kim, L. Koerner, S. Landes, W. Li, T. Magarian, A. Maruster, L. MacArthur, J. McConnell, D. Miller, J. MorcilloBlanco, M. Nelson, E. Norris, C. O'Dell., A. Peterson, M. Santiago, K. Uppstrom, B. Van Allen, and A. Vitz. We thank P. Rodewald for support of our banding efforts through his Master Banding Permit. We are grateful to the Franklin County Metro Parks, Columbus Recreation and Parks, the Ohio Division of Wildlife, The Nature Conservancy, the City of Bexley, Gahanna Parks and Recreation, and private landowners for their cooperation and access to sites. Sound recordings of Acadian Flycatchers used for capturing individuals were provided by the Borror Laboratory of Bioacoustics, Ohio State University, Columbus, Ohio, all rights reserved. T. Grubb, T. Waite, and two anonymous reviewers provided comments that improved this manuscript.
Literature Cited

Ahlering, M. A., and J. Faaborg. 2006. Avian habitat management meets conspecific attraction: If you build it, will they come? Auk 123:301-312.

Allison, P. D. 1999. Logistic regression using the SAS system: theory and applications. SAS Institute, Cary, North Carolina, USA.

Bakermans, M. H., and A. D. Rodewald. 2006. Scaledependent habitat use of Acadian Flycatcher (Empidonax virescens) in central Ohio. Auk 123:368-382.

Betts, M. G., A. S. Hadley, N. Rodenhouse, and J. J. Nocera. 2008. Social information trumps vegetation structure in breeding-site selection by a migrant songbird. Proceedings of the Royal Society B 275:2257-2263.

Biro, P. A., and J. A. Stamps. 2008. Are animal productivity traits linked to life-history productivity? Trends in Ecology and Evolution 23:361-368.

Blackmer, A. L., R. A. Mauck, J. T. Ackerman, C. E. Huntington, G. A. Nevitt, and J. B. Williams. 2005. Exploring individual quality: basal metabolic rate and reproductive performance in storm-petrels. Behavioral Ecology 16:906-913.

Burhans, D. E., and F. R. Thompson III. 2006. Songbird abundance and parasitism differ between urban and rural shrublands. Ecological Applications 16:394-405.

Campomizzi, A. J., J. A. Butcher, S. L. Farrell, A. G. Snelgrove, B. A. Collier, K. J. Gutzwiller, M. L. Morrison, and R. N. Wilkins. 2008. Conspecific attraction is a missing component in wildlife habitat modeling. Journal of Wildlife Management 72:331-336.

Chalfoun, A. D., and T. E. Martin. 2007. Assessments of habitat preferences and quality depend on spatial scale and metrics of fitness. Journal of Applied Ecology 44:983-992.

Chamberlain, D. E., A. R. Cannon, M. P. Toms, D. I. Leech, B. J. Hatchwell, and K. J. Gaston. 2009. Avian productivity in urban landscapes: a review and meta-analysis. Ibis 151:1-18.

Doligez, B., E. Danchin, and J. Colbert. 2002. Public information and breeding habitat selection in a wild bird population. Science 297:1168-1170.

Donovan, T. M., and F. R. Thompson III. 2001. Modeling the ecological trap hypothesis: a habitat and demographic analysis for migrant songbirds. Ecological Applications 11: 871-882.

Duckworth, R. A. 2008. Adaptive dispersal strategies and the dynamics of a range expansion. American Naturalist 172:S4S17.

Fretwell, S. F., and H. L. Lucas, Jr. 1970. On territorial behavior and other factors influencing habitat distribution in birds. I. Theoretical development. Acta Biotheoretica 19:16-36.

Gates, J. E., and L. W. Gysel. 1978. Avian nest dispersion and fledgling success in field-forest ecotones. Ecology 59:871-883.

Gilroy, J. J., and W. J. Sutherland. 2007. Beyond ecological traps: perceptual errors and undervalued resources. Trends in Ecology and Evolution 22:351-356.

Gosler, A. G. 1987. Pattern and process in the bill morphology of the great tit Parus major. Ibis 129:451-476.

Gotelli, N. J., and A. M. Ellison. 2004. A primer of ecological statistics. Sinauer Associates, Sunderland, Massachusetts, USA.

Grandmaison, D. D., and G. J. Niemi. 2007. Local and landscape influence on red-winged blackbird (Agelaius phoeniceus) nest success in Great Lakes coastal wetlands. Journal of Great Lakes Research 33:292-304.

Grant, B. R. 2003. Evolution in Darwin's finches: a review of a study on Isla Daphne Major in the Galapagos Archipelago. Zoology 106:255-259.

Hosmer, D. L., and S. Lemeshow. 1989. Applied logistic regression. John Wiley, New York, New York, USA.

Johnson, M. D. 2007. Measuring habitat quality: a review. Condor 109:489-504. 
Jonart, L. M., G. E. Hill, and A. V. Badyaev. 2007. Fighting ability and motivation: determinants of dominance and contest strategies in females of a passerine bird. Animal Behaviour 74:1675-1681.

Kokko, H., and W. J. Sutherland. 2001. Ecological traps in changing environments: ecological and evolutionary consequences of a behaviorally mediated Allee effect. Evolutionary Ecology Research 3:537-551.

Kristan, W. B. 2003. The role of habitat selection behavior in population dynamics: source-sink systems and ecological traps. Oikos 103:457-468.

Lewis, S., S. Wanless, D. A. Elston, M. D. Schultz, E. Mackley, M. Du Toit, J. G. Underhill, and M. P. Harris. 2006. Determinants of quality in a long-lived colonial species. Journal of Animal Ecology 75:1304-1312.

Marra, P. P., K. A. Hobson, and R. T. Holmes. 1998. Linking winter and summer events in a migratory bird using stable carbon isotopes. Science 282:1884-1886.

McNamara, J. M., and A. I Houston. 1996. State-dependent life histories. Nature 381:215-221.

Moyes, K., B. J. T. Morgan, A. Morris, S. J. Morris, T. H. Clutton-Brock, and T. Coulson. 2009. Exploring individual quality in a wild population of red deer. Journal of Animal Ecology 78:406-413.

Murphy, M. T. 2007. Lifetime reproductive success of female Eastern Kingbirds (Tyrannus tyrannus): influence of lifespan, nest predation, and body size. Auk 124:1010-1022.

Nakagawa, S. 2004. A farewell to Bonferroni: the problem of low statistical power and publication bias. Behavioral Ecology 15:1044-1045.

Neto, M. M., and A. G. Gosler. 2005. Breeding biology of the Savi's Warbler Locustella luscinoides in Portugal. Ardea 93: 89-100.

Nol, E., and N. M. Smith. 1987. Effects of age and breeding experience on seasonal reproductive success in the song sparrow. Journal of Animal Ecology 56:301-313.

Olendorf, R., and S. K. Robinson. 2000. Effectiveness of nest defense in the Acadian Flycatcher Empidonax virescens. Ibis 142:365-371.

Ortego, J., G. Calabuig, P. J. Cordero, and J. M. Aparicio. 2007. Egg production and individual genetic diversity in lesser kestrels. Molecular Ecology 16:2383-2392.

Pidgeon, A. M., V. C. Radeloff, and N. E. Mathews. 2006. Contrasting measures of fitness to classify habitat quality for the black-throated sparrow (Amphispiza bilineata). Biological Conservation 132:199-210.

Preault, M., O. Chastel, F. Cezilly, and B. Faivre. 2005. Male bill colour and age are associated with parental abilities and breeding performance in blackbirds. Behavioral Ecology and Sociobiology 58:497-505.
Pulliam, H. R., and B. J. Danielson. 1991. Sources, sinks, and habitat selection: a landscape perspective on population dynamics. American Naturalist 137:S50-S66.

Rodenhouse, N. L., T. W. Sherry, and R. T. Holmes. 1997. Site-dependent regulation of population size: a new synthesis. Ecology 78:2025-2042.

Rodewald, A. D. In press. Evaluating factors that guide avian community response to urbanization. Studies in Avian Biology.

Rodewald, A. D., and M. H. Bakermans. 2006. What is the appropriate paradigm for riparian forest conservation? Biological Conservation 128:193-200.

Rodewald, A. D., and D. P. Shustack. 2008. Urban flight: understanding individual and population-level responses of Nearctic-Neotropical migratory birds to urbanization. Journal of Animal Ecology 77:83-91.

SAS Institute. 2002. SAS/STAT Software 9.1. SAS Institute, Cary, North Carolina, USA.

Schlaepfer, M. A., M. C. Runge, and P. W. Sherman. 2002. Ecological and evolutionary traps. Trends in Ecology and Evolution 17:474-480.

Schluter, D., and J. N. M. Smith. 1986. Natural selection on beak and body size in the song sparrow. Evolution 40:221-231.

Shaffer, T. L. 2004. A unified approach to analyzing nest success. Auk 121:526-540.

Shustack, D. P. 2008. Reproductive timing of passerines in urbanizing landscapes. Dissertation. Ohio State University, Columbus, Ohio, USA.

Shustack, D. P., and A. D. Rodewald. 2008. Understanding demographic and behavioral mechanisms that guide responses of Neotropical migratory birds to urbanization: a simulation approach. Avian Conservation and Ecology $3(2): 2$.

Shustack, D. P., and A. D. Rodewald. 2010. Attenuated nesting season of the Acadian Flycatcher (Empidonax virescens) in urban forests. Auk 127:421-429.

Smith, R. J., and F. R. Moore. 2005. Arrival timing and seasonal reproductive performance in a long-distance migratory landbird. Behavioral Ecology and Sociobiology 57:231-239.

Tomiuk, J., M. Stauss, G. Segelbacher, J. Fietz, J. Kompf, and L. Bachmann. 2007. Female genetic heterogeneity affects the reproduction of great tits (Parus major L., 1758) in lowquality woodlands. Journal of Zoological Systematics and Evolutionary Research 45:144-150.

Van Horne, B. 1983. Density as a misleading indicator of habitat quality. Journal of Wildlife Management 47:893-901.

Ward, M. P., and S. Schlossberg. 2004. Conspecific attraction and the conservation of territorial songbirds. Conservation Biology 18:519-525.

\section{APPENDIX A}

Parameter estimates relating individual morphology variables and urbanization to the number of fledglings produced over the season (negative binomial regression) and the natural logarithm of day of the year of clutch initiation (least-squares regression) (Ecological Archives A020-074-A1).

\section{APPENDIX B}

Parameter estimates relating individual morphology variables and urbanization to the probability of nest survival and the probability of renesting within a season (Ecological Archives A020-074-A2).

\section{APPENDIX C}

Parameter estimates relating individual morphology variables and urbanization to the probability of resighting an individual in a future year and the number of nesting attempts made within a season (Ecological Archives A020-074-A3). 